\title{
Rhithrogena ayadi n. sp., Ephéméroptère nouveau du Moyen Atlas marocain (Heptageniidae)
}

\author{
M. Dakkil \\ A.G.B. Thomas 2
}

Mots clés : Ephemeroptera, Heptageniidae, nouvelle espèce, source, Maroc.

L'imago $\sigma$ de Rhithrogena ayadi n. sp., récoltée dans une source du Moyen Atlas (Maroc) est décrite et figurée.

Rhithrogena ayadi n. sp., a new mayfly from the moroccan Moyen Atlas (Ephemeroptera, Heptagenildae).

Keywords : Ephemeroptera, Heptageniidae, new species, spring, Morocco.

The $\sigma$ imagine of Rhithrogena ayadi $n . s p$. found in a spring in the Moyen Atlas (Morocco), is described and illustrated.

Signalé d'Algérie (Biskra) dès la fin du siècle dernier (Eaton, 1899), le gen re Rhithrogena est cependant très mal connu en Afrique du nord : il n'a fait que récemment l'objet d'une première étude taxonomique, avec la description de $R h$. ourika du Haut Atlas (Thomas et Mohati, 1985). Par manque total d'imagos $\sigma$, toutes les autres citations n'ont en effet pas dépassé le niveau générique. Or, il apparait que les larves de Rhithrogena sont souvent abondantes en Afrique du Nord, mais assez localisées. L'aire de répartition du genre recouvre sans doute une grande partie du Maghreb puisque l'un de nous (A.T.) a pu étudier du matériel de Kroumirie (M. Boumaiza leg., Tunis).

Le nombre total des espèces nord-africaines paraît pouvoir être estimé au minimum à trois ou quatre, ne serait-ce qu'en raison des différences considérables observées sur les parametres écologiques. En particulier, l'une des espèces se montre nettement potamophile (Dakki et El Agbani, 1983). A l'inverse, lors de la prospection de sources du Rif et du Moyen

1. Département de Zoologie et Ecologie animale, Inst itut Scientifique de Rabat, B.P. 703, Rabat-Agdal, Maroc.

2. Laboratoire d'Hydrobiologie, UA 695 du C.N.R.S., Universite Paul Sabatier, 118 route de Narbonne, 31062 Toulouse Cédex. France.
Atlas (Giudicelli et Dakki, 1984), ont été récoltés des adultes $\sigma$ d'une espèce nouvelle, apparemment crénobionte stricte. Elle est dédiée à $\mathrm{M}$. Ayad, technicien à l'Institut Scientifique de Rabat.

\section{Rhithrogena ayadi n. sp.}

IMAGo o (conservation en alcool à $70^{\circ}$ ).

Tête.

Face brun grisâtre ; vertex brun moyen à foncé. Ocelles gris clair, les mamelons ocellaires cerclés de brun noirâtre. Antennes brunes. Le pli transversal qui surmonte l'insertion de chaque antenne est de la mème couleur que la face, alors qu'il est brun plus foncé chez $R h$. ourika. Yeux rosâtres, sans bande latérale individualisée, plus clairs que chez $R h$. ourika.

Thorax.

Prothorax. Tergite et sternite brun foncé.

Méso et métathorax. Partie centrale du scutum brun clair, nettement contrastée par rapport aux régions antérieure et postérieure, d'un brun foncé comme le tergite métathoracique. Sternite mésothoracique brun moyen, plus foncé en arrière de $\mathbf{P} 2$. Sternite métathoracique clair, brun grisâtre. Il 

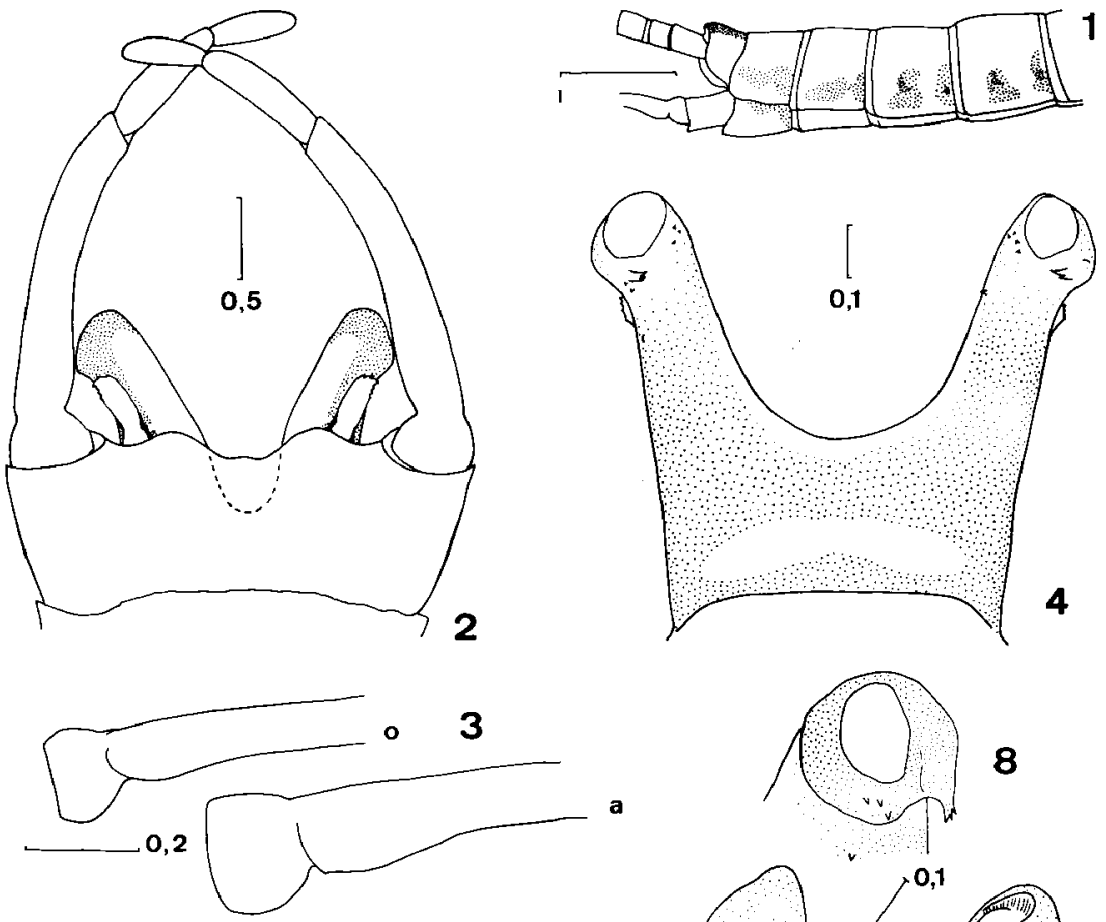

a
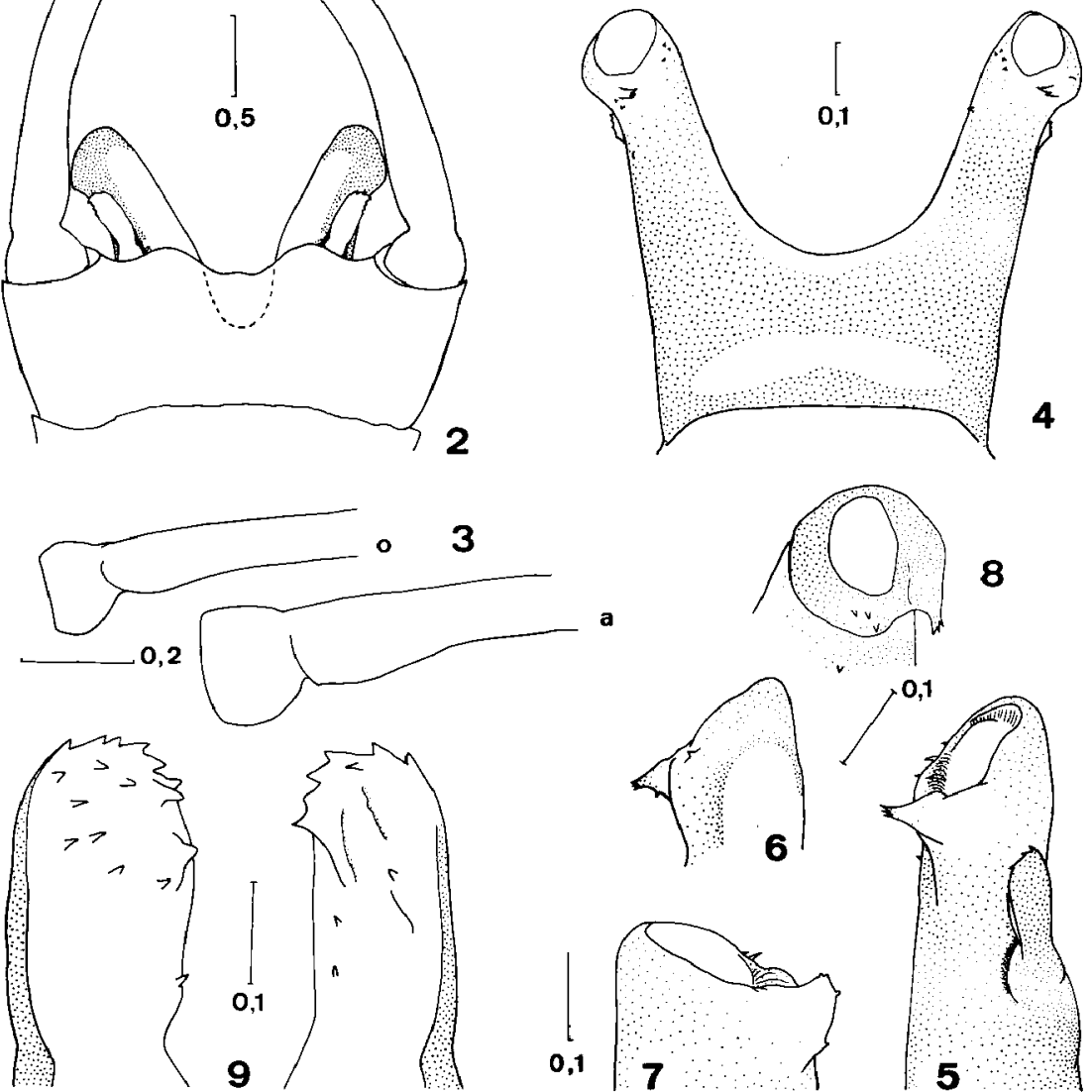

8

Fig. 1 à 9 : imago ơ de Rhithrogena ayadi $\mathrm{n}$. sp. Echelle en $\mathrm{mm}$.

1 : vue latérale de l'abdomen. $2:$ styligère, styles et lobes péniens en vue ventrale. 3 : région proximale des styles $(\mathrm{o}=$ Rh. ourika Thomas et Mohati ; $\mathrm{a}=$ ayadi $) .4$ : pénis en vue dorsale. 5 à $8:$ apex des lobes péniens $(5:$ profil externe; $6:$ profil interne ; $7:$ vue latéro-ventrale; $8:$ vue caudale). $9:$ titillateurs. 
n'existe aucune trace des traînées de pigment foncé, violacé, partant de la base de l'aile antérieure en direction de la première coxa, observées chez $R h$. ourika. Coxas et trochanters brun clair. Fémurs 2 et 3 brun jaunâtre sale, portant une tache médiane externe brun violacé intense, beaucoup plus développée que chez $R h$. ourika. La face interne des fémurs porte elle aussi une tache médiane de même couleur mais plus petite (totalement absente chez $R h$. ourika). Tibias gris jaunâtre clair, tarses brun moyen sale. Ailes antérieures entièrement claires depuis la base, à l'exception de l'aire ptérostigmatique enfumée, traversée par des nervures très peu ou pas ramifiées. Par rapport à $R h$. ourika, la membrane est cependant moins hyaline et les nervures transverses jusqu'à R2 sont étroites et dépourvues de pigment violacé : toute la nervation est brum moyen. Ailes postérieures hyalines, à fine nervation brun jaunâtre très claire.

\section{Abdomen.}

Couleur de base brun jaunâtre terne, à peine plus foncée dans la région dorsale postérieure des tergites. Ces derniers portent, vers le milieu, près des bords latéraux (fig. 1) une tache brune plus ou moins cuivrée et assez petite. Une autre tache, plus ou moins constante, peut se trouver près des coins antérieurs des tergites. Ces deux taches sont coalescentes sur les segments 8 et 9 . Sternites brun jaunâtre terne avec deux traces brunes antérieures obliques en tirets. Bords antérieur et latéraux du ge sternite bruns. Dernier tergite portant deux taches brun foncé, chacune dans le prolongement d'un cerque. Cerque brun foncé s'éclaircissant progressivement vers l'apex.

Genitalia.

Styligère jaune, la base des styles jaune à brune portant deux protubérances jaunes (brun foncé chez $R h$. ourika), bien marquées et nettement espacées (fig. 2). Styles brun grisâtre assez foncé et massifs ; le premier article est fortement élargi à la base, du côté interne et sur la face ventrale, immédiatement après l'articulation ( $\mathrm{fg}$. 3). Lobes péniens plus longs et formant entre eux un angle plus aigu que chez
Rh. ourika (fig. 2 et 4). Apex des lobes tronqué dorsalement, portant une ou plusieurs petites épines, irrégulièrement disposées, du côté interne et un fort crochet latéro-dorsal à plusieurs pointes (fig. 4 à 7 ). Titillateurs larges, à apex arrondi et multidenticulé, avec aussi quelques épines sur la surface ventrale (fig. 9).

Taille.

Elle est voisine de celle de $R h$. ourika.

Longueur de l'aile $\left(\sigma^{\circ}\right): 10,2$ à $11,0 \mathrm{~mm}$. Longueur des cerques : 21 à $26 \mathrm{~mm}$.

\section{Matériel examiné.}

3 imagos $\sigma$ (l'holotype et un paratype sont déposés à l'Institut Scientifique de Rabat, un second paratype dans la collection A. Thomas, Toulouse) capturées en juillet 1982 à la source Amane Imelallen (altitude : $2150 \mathrm{~m}$ ). Cette source est située sur le flanc nord-ouest du massif du Bou Iblane (bassin de l'oued Zloul, Moyen Atlas), à environ $3 \mathrm{~km}$ à l'est du refuge de Taffert.

Les larves de $R h$, ayadi n'ont été rencontrées que dans cette source, la plus élevée des 12 prospectées par Giudicelli et Dakki (1984), mais elles y sont très abondantes. La température maximale de l'eau a été relevée en juillet $1981: 15^{\circ} \mathrm{C}$. Le débit est de l'ordre de $5 \mathrm{l} / \mathrm{s}$.

Travaux cités

Dakki (M.) et El Agbani (M.A.). 1983. - Ephéméroptères d'Afrique du nord. 3, éléments pour la connaissance de la faume marocaine. Bull. Inst. scient. Rabat, $7 ; 115-126$.

Eaton (A.E.). 1899. - List of Ephemeridae hitherto observed in Algeria with localities. Entomologist's mon. Mag., $35: 45$.

Giudicelli (J.) et Dakki (M.). 1984. - Les sources du Moyen Atlas et du Rif (Maroc) : faunistique (description de deux especes nouvelles de Trichoptères), écologie, intérêt biogéographique. Bijdr. Dierk., 54 (1) : 83-100.

Thomas (A.G.B.) et Mohati (A.). 1985. - Rhithrogena ourika n. sp., Epheméroptère nouveau du Haut Atlas marocain (Heptageniidae). Annls Limnol., 21 (2) : 145-148. 